\title{
Hidden danger of COVID-19 outbreak: evaluation of subclinical myocardial dysfunction in patients with mild symptoms
}

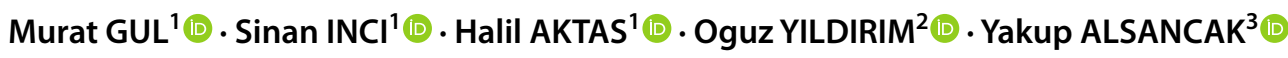

Received: 31 March 2021 / Accepted: 15 June 2021 / Published online: 22 June 2021

(c) The Author(s), under exclusive licence to Springer Nature B.V. 2021

\begin{abstract}
The COVID-19 infection, which is caused by the novel coronavirus SARS-CoV-2, has rapidly emerged as a global public health issue. Cardiac complications secondary to this infection are common and associated with mortality. This study aimed to evaluate whether subclinical myocardial dysfunction exists in non-hospitalized mildly symptomatic COVID-19 patients using left ventricular global longitudinal strain (LVGLS). In this cross-sectional, single-center study, data were collected from non-hospitalized mildly symptomatic COVID-19 patients between January 01 and February 01, 2021. Fifty (26 male, 24 female) COVID-19 patients and 50 age- and sex-matched healthy volunteers were included in the study. Apical four-, three-, and two-chamber images were analyzed longitudinally by conventional methods and speckle tracking echocardiography (STE) for left ventricle functions. The mean age of the COVID-19 patients was $39.5 \pm 8.96$, and 52\% of them were male. The most prevalent presenting symptoms were fever [in $34(68 \%)$ ], asthenia [in $30(60 \%)$ ], loss of appetite [in 21 (42\%)], myalgia [in $20(40 \%)$ ], and cough [in $13(26 \%)$ ]. Plasma levels of C-reactive protein (CRP) were significantly higher in the COVID-19 patients than in the controls $(10.84 \pm 12.44$ vs. $4.50 \pm 2.81, \mathrm{p}<0.001)$. There was no significant difference between the groups in terms of standard echocardiography and Doppler parameters ( $p>0.05)$. Left ventricular longitudinal strain and strain velocity parameters were significantly decreased in COVID-19 patients compared to healthy individuals. LV-GLS values $(-21.72 \pm 3.85 \%$ vs. $-23.11 \pm 4.16 \%$; $p=0.003)$ were significantly lower in COVID-19 patients compared with the healthy controls. Mildly symptomatic COVID-19 patients also have subclinical myocardial dysfunction similar to hospitalized patients. STE has the potential for detecting subclinical LV systolic dysfunction, and can provide useful information regarding cardiac status in mildly symptomatic COVID-19 population.
\end{abstract}

Keywords COVID-19 $\cdot$ Speckle tracking echocardiography $\cdot$ Subclinical myocardial dysfunction $\cdot$ Global longitudinal strain

Murat GUL

drmuratgul68@gmail.com

Sinan INCI

doktorsinaninci@gmail.com

Halil AKTAS

halilaktas_85@hotmail.com

Oguz YILDIRIM

oguzyldrm29@gmail.com

Yakup ALSANCAK

dryakupalsancak@gmail.com

1 Department of Cardiology, School of Medicine, Aksaray University, 68100 Aksaray, Turkey

2 Department of Cardiology, Aksaray Education and Research Hospital, Aksaray, Turkey

3 Department of Cardiology, Meram School of Medicine, Necmettin Erbakan University, Konya, Turkey

$\begin{array}{ll}\text { Abbreviations } \\ \text { AFI } & \text { Automated function imaging } \\ \text { AVC } & \text { Aortic valve closure } \\ \text { CRP } & \text { C-reactive protein } \\ \text { DT } & \text { Deceleration time } \\ \text { GLS } & \text { Global longitudinal strain } \\ \text { GLSR } & \text { Global longitudinal strain rate } \\ \text { IVRT } & \text { Isovolumetric relaxation time } \\ \text { IVS } & \text { Interventricular septum diameter } \\ \text { LSRA } & \text { Longitudinal strain rate diastolic late filling } \\ \text { LSRE } & \text { Longitudinal strain rate diastolic early filling } \\ \text { LSRS } & \text { Longitudinal strain rate systolic } \\ \text { LVEDD } & \text { Left ventricle end-diastolic diameter } \\ \text { LVEF } & \text { Left ventricular ejection fraction } \\ \text { LVESD } & \text { Left ventricle end-systolic diameter } \\ \text { LVGLS } & \text { Left ventricular global longitudinal strain } \\ \text { M-mode } & \text { Motion-mode }\end{array}$




$\begin{array}{ll}\text { PW } & \text { Posterior wall } \\ \text { ROI } & \text { Region of interest } \\ \text { RT-PCR } & \text { Real-time polymerase chain reaction } \\ \text { STE } & \text { Speckle tracking echocardiography } \\ \text { TDI } & \text { Tissue Doppler imaging } \\ \text { 2D } & \text { Two-dimensional }\end{array}$

\section{Introduction}

The COVID-19 infection, which is caused by the novel coronavirus SARS-CoV-2, has rapidly emerged as a global public health issue. As of January 5, 2021, infected patients were present in 222 countries, and there were $>84$ million confirmed cases, with more than 1.8 million fatalities [1]. The clinical spectrum of COVID-19 ranges from an asymptomatic form to severe acute respiratory syndrome and even death $[2,3]$. The proportion of patients who remained asymptomatic throughout the infection was approximately $20 \%$ of all confirmed cases [4]. Approximately $80 \%$ of symptomatic patients have experienced a mild clinical course, defined as dry cough, sore throat, low-grade fever, or malaise [5]. Although the clinical manifestations are mainly respiratory, numerous studies have reported that cardiac complications are common (20-25\%) and associated with mortality $[6,7]$.

The COVID-19 could adversely affect the cardiovascular system by direct myocardial injury, severe systemic inflammatory response, hypoxia, or inflammatory plaque rupture [8]. Primary cardiac manifestations include acute myocardial infarction, fulminant myocarditis, heart failure, malign arrhythmias, and cardiogenic shock [9]. In a single-center study, $19 \%$ of hospitalized patients due to COVID-19 experienced a cardiac injury, and these patients had worse clinical outcomes [10]. Moreover, long-term cardiac sequelae have been identified in patients who recently recovered from COVID-19, regardless of the severity and overall course of the acute illness [11]. These findings suggest that cardiac damage may be observed even in patients without evident myocardial involvement during acute infection. Therefore, early diagnosis of cardiac involvement is crucial for vigilant monitoring and timely treatment of high-risk patients.

Two-dimensional (2D) echocardiography is the most preferred imaging modality for cardiovascular diseases, but it does not show intrinsic myocardial properties. Speckle tracking echocardiography (STE) has emerged as an echocardiographic parameter predicting left ventricular myocardial injury and future cardiac events [12]. Previous studies have shown that STE evaluates myocardial dysfunction and subclinical myocardial impairment earlier and more precisely than conventional echocardiography [13]. Further, myocardial strain imaging by STE has also provided prognostic information in patients hospitalized due to COVID-19
[13]. However, there are limited data on whether COVID-19 has any cardiac effects in non-hospitalized mildly symptomatic patients. We, therefore, aimed to evaluate whether subclinical myocardial dysfunction exists in non-hospitalized mildly symptomatic COVID-19 patients using left ventricular global longitudinal strain (LVGLS).

\section{Methods}

\section{Design and study population}

This is a cross-sectional study that was conducted in a single tertiary healthcare center between January 1 and February 1, 2021. Exclusion criteria were presence of coronary or peripheral vascular diseases, heart failure with reduced ejection fraction $(\mathrm{EF}<50 \%)$, segmental wall-motion abnormalities of the left ventricle, structural heart disease, moderate or severe valvular heart disease, pericarditis or massive pericardial effusion, abnormal electrocardiography findings (STsegment changes, conduction disturbances, atrial fibrillation, etc.), systolic pulmonary artery pressure (PASP) $>40 \mathrm{mmHg}$ or known pulmonary arterial hypertension, diabetes mellitus, hypertension, chronic kidney disease, body mass index (BMI) $>35 \mathrm{~kg} / \mathrm{m}^{2}$, chronic obstructive pulmonary diseases, restrictive pulmonary disease, radiological evidence indicating pulmonary involvement of COVID-19, connective tissue disorders, malignancy, individuals with systemic and metabolic diseases that could adversely affect the cardiac functions, and smoking. Patients younger than 18 years of age and subjects with poor image quality were also excluded from the study.

Only confirmed COVID-19 patients who had mild symptoms for more than a week and no evidence of pneumonia and/or cardiac injury were included in the study. Mildly symptomatic patients were defined as subjects with mild subjective symptoms such as fever, headache, sore throat, and nasal congestion but without clinical or radiological evidence of pneumonia [14]. Cardiac injury was defined as an elevation of troponin levels above the 99th percentile upper reference limit, which is the most commonly reported cardiac abnormality in COVID-19 [15]. The diagnosis of COVID-19 was confirmed by real-time polymerase chain reaction (RT-PCR) testing of nasopharyngeal samples.

A total of 58 consecutive patients who had been decided on home-based management were included in the study. Patients diagnosed with COVID-19 infection underwent comprehensive echocardiographic evaluation within $24 \mathrm{~h}$ of admission. Echocardiography was performed during the index evaluation and they were discharged from the hospital after the necessary examinations were made about their diseases. All patients were followed up via daily phone interviews to evaluate the possibility of precipitous 
clinical deterioration. Body temperature, respiration pattern, and other symptoms were routinely assessed by self-monitoring and reporting. In case of the emergence of new symptoms, patients were evaluated by physicians, and patients requiring hospitalization and/or advanced medical therapy $(n=6)$ were excluded from the study. Two patients who refused to continue follow-up visits were also excluded. Finally, the data of the remaining 50 patients were analyzed. We also enrolled age and gender-matched fifty healthy individuals as the control group. Individuals who had no clinical complaints for the last 1 week, and no laboratory abnormalities and whose RT-PCR testing of nasopharyngeal samples performed within $24 \mathrm{~h}$ were negative were taken as the control group. Patients' data, including demographics, medical history, and laboratory examinations were also collected and analyzed.

\section{Transthoracic echocardiography}

Echocardiographic examinations were performed by EPIQ 7 digital ultrasound scanner (Philips Medical System, USA) in the left lateral decubitus position from multiple windows. All measurements were taken by two experienced cardiologists who were blinded to the clinical status of the patients. A single-derivation electrocardiogram was simultaneously recorded during the examination. Standard echocardiographic images (parasternal long-axis, parasternal short-axis, apical-two chamber, apical three-chamber, and apical four-chamber views) were captured and evaluated by motion mode (M-mode), 2D, continuous stream Doppler, and tissue Doppler imaging (TDI) according to the American Echocardiography Association's criteria [16]. Left ventricle end-diastolic diameter (LVEDD; mm), left ventricle end-systolic diameter (LVESD; mm), interventricular septum diameter (IVS; $\mathrm{mm}$ ) at end-diastole, and posterior wall diameter (PW) at end-diastole were obtained from the M-mode echocardiographic tracing under the guide of 2D. The left ventricular ejection fraction (LVEF; \%) was measured using the biplane modified Simpson method (diastolic volume - systolic volume)/ (diastolic volume) as recommended by current guidelines (17). Diastolic functions were evaluated by measurement of transmitral flow parameters including the early (E) and late (A) diastolic filling velocities, the E/A ratio, deceleration time (DT), and isovolumetric relaxation time (IVRT) from an apical four-chamber view with the sample volume sited at the tip of the mitral leaflet. Tissue Doppler velocities were measured from the septal and lateral annulus in apical four-chamber view. The peak systolic $(\mathrm{Sm})$, early diastolic (Em), and late diastolic velocity (Am) were also measured. The values were determined by averaging three subsequent beats.

\section{Strain and strain rate measurements}

Left ventricular "Global Longitudinal Strain" (GLS), "Longitudinal Strain Rate Systolic" (LSRS), "Longitudinal Strain Rate Diastolic Early Filling" (LSRE), and "Longitudinal Strain Rate Diastolic Late Filling" (LSRA) parameters were measured using automated function imaging (AFI) [17]. 2D echocardiographic images were constructed from apical 4-chamber (4C), 3-chamber (3C), and apical 2-chamber (2C) images and left ventricle images were recorded digitally. The imaging that best defined the endocardial margin was employed to provide the records. The average frame rate of $60 / \mathrm{s}(50-80 / \mathrm{sec})$ and three cardiac beats were captured for each measurement.

The initial phase in strain analysis is determining the aortic valve closure (AVC) time by employing conventional or AFI methods. Aortic valve closure was identified at baseline and at each grade of procedure in the apical 3-chamber view. We determined the AVC time automatically with the help of the software at the end of the T wave. The accuracy of the timing was validated by identifying the AVC visually using the B-mode image, and before finalizing its electrocardiographic location, the necessary adjustments were done. We started measurement from the basal septum, manually placing 6 or 7 equally distributed endocardial points. Using these points, the 2D speckle tracking algorithm followed the endocardium as the region of interest (ROI) to determine the wall thickness along the heart cycle. ROI consisted of three concentric lines representing the endocardial and epicardial margins and the middle myocardial layer. The required settings were made to match the myocardial thickness, excluding the pericardium. The left ventricle, 4-chamber, 3-chamber, 2-chamber images were analyzed using STE. Furthermore, strain parameters of each segment were evaluated by employing the AFI protocol (Fig. 1).

\section{Statistical analysis}

In order to calculate the sample size of the study, LVGLS (\%) was taken as the primary outcome variable. Group sample sizes were found to be 49 and 49 to achieve $90.4 \%$ power to detect a difference of $2 \%$ between both groups' means values and with a significance level (alpha) of 0.05 using a two-sided two-sample t-test.

The data were summarized as mean \pm standard deviation for continuous variables, and frequencies (percentiles) for categorical variables. Student's t-test or Mann Whitney U test was used for independent group comparisons, depending on the distributional properties of the data. Chi-Square test was used for proportions, and its counterpart Fisher's Exact test was used when the data were sparse. To evaluate reliability, two-way random, absolute agreement intraclass correlation coefficient (ICC) was used for randomly selected 


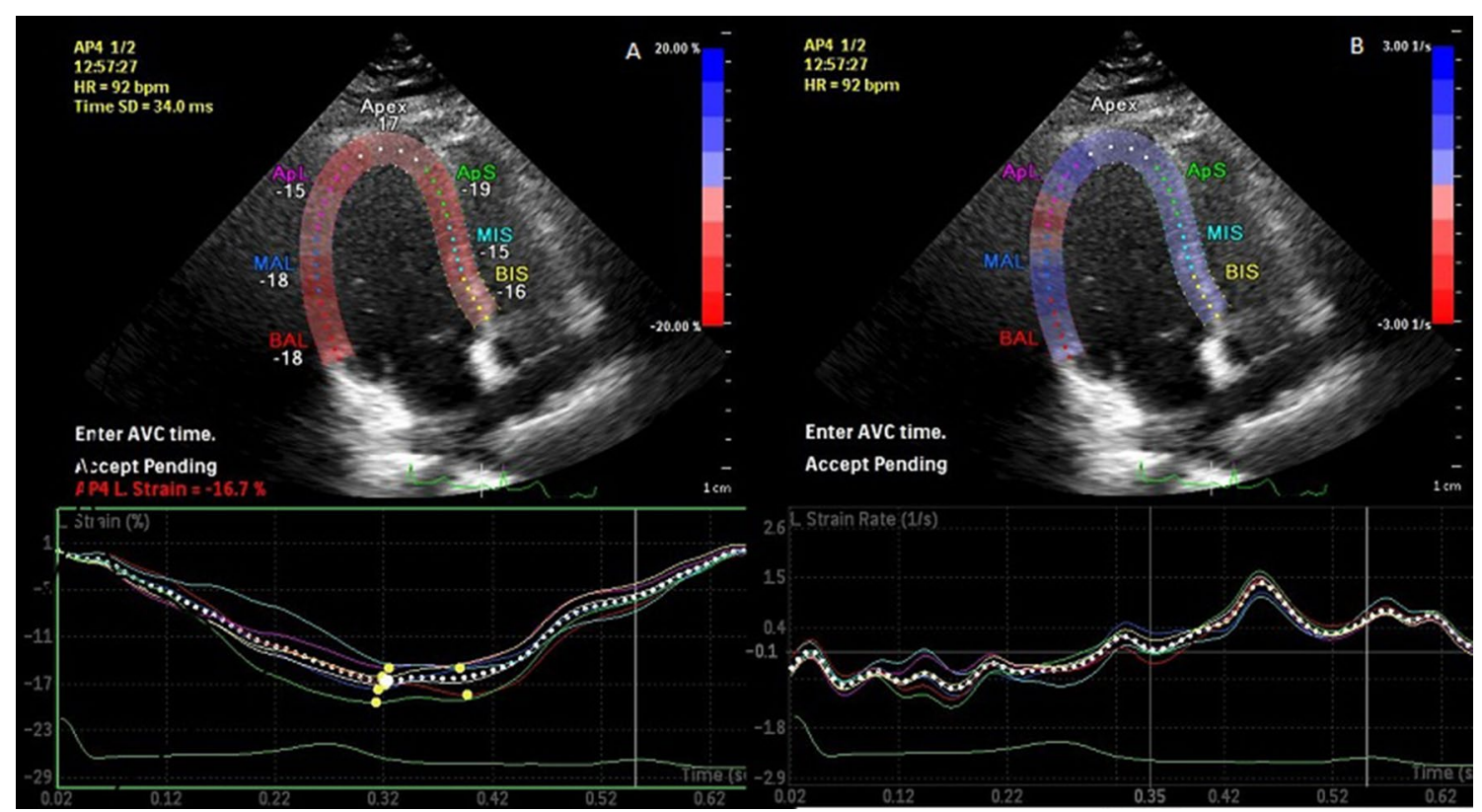

Fig. 1 Longitudinal strain (A) and strain rate (B) measurement with two-dimensional speckle tracking echocardiography in an apical four-chamber view

20 patients on the parameters GLS and GLS. All analyses were performed with IBM SPSS Statistics for Windows, Version 20.0. A p value $<0.05$ was considered statistically significant.

\section{Results}

A total of 100 patients were included in this study. The mean age of the COVID-19 group was $39.5 \pm 8.96$, and $52 \%$ of them were male. The median duration from the first symptoms to hospital admission was 8.5 days (IQR 6.5-11). The most prevalent presenting symptoms were fever [in 34 patients $(68 \%)$ ], asthenia [in $30(60 \%)$ ], loss of appetite [in $21(42 \%)$ ], myalgia [in $20(40 \%)$ ], and cough [in 13 (26\%)]. Clinical, laboratory, and demographic data of the study population are summarized in Table 1 . The COVID19 group and controls were similar regarding age, gender, BMI, heart rate, creatinine, glucose, white blood cell, hemoglobin, thrombocyte, lymphocyte, fibrinogen, ferritin, lactate dehydrogenase, D-Dimer, systolic blood pressure, and diastolic blood pressure $(\mathrm{p}>0.05)$. Plasma levels of C-reactive protein (CRP) were significantly higher in the COVID-19 group than in the controls $(10.84 \pm 12.44$ vs. $4.50 \pm 2.81$, $\mathrm{p}<0,001)$.

In conventional echocardiographic measurements, LVEDD, LVESD, IVS, PW, LVEF, E, A, DT, E/A ratio, and IVRT were also similar between the groups. When TDI parameters measured from the lateral mitral annulus were compared, there was no statistically significant difference in Em, Sm, and Am values between groups (p>0.05) (Table 2).

Left ventricular longitudinal strain and strain velocity parameters of the study population are shown in Table 3. The mean LVGLS was significantly low in COVID-19 patients compared to healthy controls $(-21.72 \pm 3.85 \%$ vs. $-23.11 \pm 4.16 \%, \mathrm{p}=0.003)$. The global LSRS was also lower in the COVID-19 group than controls $(-1.21 \pm 0.42$ vs. $-1.76 \pm 0.96 / \mathrm{sec}, \mathrm{p}<0.001)$. Both global LSRE $(1.92 \pm 0.43$ vs. $2.34 \pm 0.95 / \mathrm{sec}, \mathrm{p}=0.05)$, and global LSRA $(0.80 \pm 0.23$ vs. $1.05 \pm 0.49 / \mathrm{sec}, \mathrm{p}=0.001)$ values were significantly decreased in the COVID-19 group compared to controls.

Twenty patients were selected randomly to assess interobserver and intra-observer variability. Inter-observer variability for GLS and GLSR was less than $3.7 \%$ and $4.5 \%$, respectively. Intra-observer variability was found less than $4.5 \%$.

\section{Discussion}

To our knowledge, this is the first study on LVGLS changes in patients with mild symptomatic COVID-19 infection. Our study revealed that: (i) left ventricular longitudinal systolic strain and strain rate parameters were significantly reduced in patients with COVID-19 compared to healthy controls; (ii) CRP levels were higher in the COVID-19 group compared to the controls; (iii) conventional echocardiography parameters were similar between COVID-19 and healthy 
Table 1 Clinical, laboratory, and demographic data of the study population

\begin{tabular}{|c|c|c|c|}
\hline Parameters & $\begin{array}{l}\text { COVID-19 group } \\
(\mathrm{n}=50)\end{array}$ & $\begin{array}{l}\text { Control group } \\
(n=50)\end{array}$ & $\mathrm{p}$ value \\
\hline Age (year) & $39.5 \pm 8.96$ & $37.2 \pm 8.04$ & 0.166 \\
\hline Body Mass Index $\left(\mathrm{kg} / \mathrm{m}^{2}\right)$ & $28.11 \pm 3.43$ & $27.74 \pm 3.21$ & 0.574 \\
\hline Male $(\%)$ & $26(52)$ & $25(50)$ & 0.536 \\
\hline \multicolumn{4}{|l|}{ Presenting symptoms (\%) } \\
\hline Fever & $34(68)$ & - & \\
\hline Asthenia & $30(60)$ & - & \\
\hline Loss of appetite & $21(42)$ & - & - \\
\hline Myalgia & $20(40)$ & - & \\
\hline Cough & $13(26)$ & - & \\
\hline Heart rate $(\mathrm{bpm})$ & $71.09 \pm 9.37$ & $71.49 \pm 11.69$ & 0.851 \\
\hline Glucose (mg/dL) & $82.30 \pm 17.16$ & $84.17 \pm 14.67$ & 0.554 \\
\hline Creatinine (mg/dL) & $0.71 \pm 0.16$ & $0.72 \pm 0.15$ & 0.745 \\
\hline Hemoglobin $(\mathrm{g} / \mathrm{dL})$ & $13.86 \pm 1.37$ & $13.92 \pm 1.16$ & 0.823 \\
\hline White blood cell counts $\left(10^{6} / \mathrm{L}\right)$ & $7359.03 \pm 1679.05$ & $7125.03 \pm 1573.86$ & $0.468^{*}$ \\
\hline Lymphocytes counts $\left(10^{6} / \mathrm{L}\right)$ & $1294.14 \pm 421.06$ & $1421.07 \pm 368.07$ & 0.160 \\
\hline Thrombocyte counts $\left(10^{6} / \mathrm{L}\right)$ & $338.23 \pm 119.24$ & $366.50 \pm 118.79$ & 0.231 \\
\hline D-Dimer $(\mu \mathrm{g} / \mathrm{mL})$ & $370.86 \pm 175.54$ & $364.64 \pm 158.45$ & $0.851^{*}$ \\
\hline $\mathrm{CRP}(\mathrm{mg} / \mathrm{L})$ & $10.84 \pm 12.44$ & $4.50 \pm 2.81$ & $<0.001$ \\
\hline Fibrinogen $(\mathrm{g} / \mathrm{L})$ & $3.07 \pm 0.74$ & $2.90 \pm 0.47$ & 0.513 \\
\hline Ferritin (ng/mL) & $317.14 \pm 45.06$ & $336.84 \pm 34.09$ & 0.199 \\
\hline Lactate dehydrogenase (U/L) & $205.57 \pm 71$ & $194.79 \pm 42$ & 0.320 \\
\hline Systolic blood pressure (mmHg) & $125.84 \pm 10.44$ & $125.50 \pm 9.81$ & 0.612 \\
\hline Diastolic blood pressure $(\mathrm{mmHg})$ & $82.11 \pm 6.21$ & $81.66 \pm 6.83$ & 0.728 \\
\hline
\end{tabular}

* Represents the $\mathrm{p}$ value for Mann Whitney U test group. These findings suggest the presence of occult myocardial injury even in the mild symptomatic COVID-19 patients. STE may be a practical imaging tool for detecting subclinical myocardial dysfunctions, even when LVEF is preserved.

Cardiac complications are a notable aspect of the COVID-19 disease. Myocardial injury, heart failure, acute coronary syndrome, and thromboembolic events are the most common reported cardiac disorders in the literature [18]. Cases of fulminant myocarditis with atrial and ventricular arrhythmia have also been reported [19]. The data from Italy have reported that sudden cardiac death occurred in non-hospitalized patients with mild symptoms who were inhome quarantine [20]. These unexpected deaths have raised concerns about the cardiac involvement of COVID-19. We believe that early detection of high-risk patients is crucial, as close monitoring and timely interventions could prevent adverse cardiac events.

Cardiac involvement of COVID-19 infection may be due to multiple factors. Relevant mechanisms can be summarized as follows: (i) Cytokine storm and myocardial damage due to acute systemic inflammatory response; (ii) oxygen supply-demand imbalance of the myocardium secondary to acute respiratory failure and hypoxia; (iii) possible cardiotoxic effects of agents used in the treatment; (iv) microvascular and macrovascular thrombosis due to systemic inflammation and endothelial dysfunction; (v) tendency to arrhythmia due to hypoxia, systemic inflammation, and changes on ACE-2 signaling system; (vi) epicardial coronary plaque rupture as a result of shear stress; (vii) possibly a direct viral invasion of the myocardium $[13,21]$. In our study, we observed an impairment in longitudinal systolic strain despite normal systolic parameters measured by TDI and conventional echocardiography. The early manifestation of cardiac abnormalities is usually observed in the subendocardial layer since subendocardial fibers are more vulnerable to myocardial ischemia and inflammation [22, 23]. The CRP levels were also consistently higher in the COVID-19 patients than in healthy subjects. We speculate that subendocardial longitudinal fibers will be the first affected region before obvious cardiac manifestation due to ongoing inflammation. Therefore, the measurement of the LVGLS appears to be a reliable method in the early detection of subclinical cardiac dysfunction.

Left ventricular global longitudinal strain measured by STE is an objective and reproducible technique that measures myocardial deformation with angle independence. GLS is a more sensitive predictor of left ventricular dysfunction 
Table 2 Comparison of the conventional echocardiographic characteristics of the study population

\begin{tabular}{lccc}
\hline Parameters & $\begin{array}{l}\text { COVID-19 group } \\
(\mathrm{n}=50)\end{array}$ & $\begin{array}{l}\text { Control group } \\
(\mathrm{n}=50)\end{array}$ & p value \\
\hline IVS $(\mathrm{mm})$ & $11.36 \pm 1.31$ & $11.25 \pm 1.32$ & 0.672 \\
PW $(\mathrm{mm})$ & $10.71 \pm 1.01$ & $10.62 \pm 1.09$ & 0.687 \\
LVEDD $(\mathrm{mm})$ & $47.98 \pm 4.68$ & $47.94 \pm 4.04$ & 0.963 \\
LVESD $(\mathrm{mm})$ & $28.88 \pm 4.63$ & $29.50 \pm 3.80$ & 0.457 \\
EF $(\%)$ & $63.94 \pm 4.16$ & $64.82 \pm 3.64$ & 0.256 \\
DT $(\mathrm{msn})$ & $186.83 \pm 34.62$ & $180.62 \pm 28.78$ & $0.341^{*}$ \\
IVRT $(\mathrm{msn})$ & $80.76 \pm 24.50$ & $72.52 \pm 18.35$ & 0.057 \\
E (m/sn) & $77.69 \pm 17.04$ & $79.39 \pm 15.52$ & 0.598 \\
A $(\mathrm{m} / \mathrm{sn})$ & $61.76 \pm 9.54$ & $58.01 \pm 8.35$ & 0.056 \\
Septal anulus & & & \\
Sm $(\mathrm{cm} / \mathrm{s})$ & $10.46 \pm 3.23$ & $10.89 \pm 3.33$ & 0.163 \\
Em $(\mathrm{cm} / \mathrm{s})$ & $10.89 \pm 3.02$ & $11.09 \pm 3.04$ & 0.236 \\
Am $(\mathrm{cm} / \mathrm{s})$ & $11.69 \pm 2.39$ & $11.99 \pm 2.76$ & 0.523 \\
Lateral anulus & & & \\
Sm $(\mathrm{cm} / \mathrm{s})$ & $11.25 \pm 2.92$ & $11.79 \pm 3.04$ & 0.458 \\
Em $(\mathrm{cm} / \mathrm{s})$ & $12.36 \pm 3.09$ & $12.52 \pm 3.22$ & 0.525 \\
Am $(\mathrm{cm} / \mathrm{s})$ & $9.46 \pm 1.69$ & $9.63 \pm 1.79$ & 0.637 \\
\hline
\end{tabular}

$A$ late diastolic filling velocity, $A m$ Tissue Doppler late diastolic velocity, $D T$ deceleration time, $E$ early diastolic filling velocity, $E F$ ejection fraction, Em Tissue Doppler early diastolic velocity, IVRT isovolumetric relaxation time, IVS interventricular septum, $L A$ left atrium, $L V E D D$ left ventricle end-diastolic diameter, $L V E S D$ left ventricle end-systolic diameter, $P A S P$ systolic pulmonary artery pressure, $P W$ posterior wall, Sm Tissue Doppler peak systolic velocity

*Represents the $\mathrm{p}$ value for Mann Whitney U test

than LVEF and provides additional prognostic value to LVEF. It can also predict subclinical myocardial dysfunction before overt cardiovascular diseases, even in asymptomatic patients [24]. The diagnostic and prognostic role of STE has been demonstrated in different clinical settings such as LV hypertrophy, cardio-oncology, valvular diseases, and cardiomyopathies [25]. Lower LVGLS in large patient populations predicts future cardiac events regardless of LVEF, age, gender, and hypertension [26]. The presence of impaired longitudinal function in hospitalized COVID-19 patients has also been demonstrated previously [27]. Moreover, Janus et al. demonstrated that reduction in LVGLS is a powerful predictor of mortality in COVID-19 patients [28]. As the long-term cardiovascular complications of COVID-19 infection are not known yet, patients with preserved LVEF and reduced LVGLS may benefit from close observation.

Currently, myocardial injury due to COVID-19 is mainly defined by elevated serum troponin levels with a prevalence of $8-30 \%$ of affected patients $[29,30]$. It is associated with fatal outcomes such as respiratory failure requiring mechanical ventilation, life-threatening arrhythmias, and mortality [31]. However, autopsy-based studies reported that COVID19 cases often have cardiac fibrin microthrombi without
Table 3 Comparison of longitudinal and global strain-strain rate parameters

\begin{tabular}{lrrr}
\hline Parameters & \multicolumn{1}{l}{$\begin{array}{l}\text { COVID-19 group } \\
(\mathrm{n}=50)\end{array}$} & $\begin{array}{l}\text { Control group } \\
(\mathrm{n}=50)\end{array}$ & $\mathrm{p}$ value \\
\hline $\mathrm{S}-4 \mathrm{C}(\%)$ & $-20.96 \pm 3.85$ & $-22.91 \pm 4.21$ & 0.022 \\
$\mathrm{~S}-3 \mathrm{C}(\%)$ & $-21.52 \pm 3.89$ & $-22.98 \pm 4.19$ & 0.058 \\
$\mathrm{~S}-2 \mathrm{C}(\%)$ & $-21.67 \pm 3.80$ & $-23.29 \pm 4.12$ & 0.017 \\
GLS $(\%)$ & $-21.72 \pm 3.85$ & $-23.11 \pm 4.16$ & 0.003 \\
SRS-4C, 1/s & $-1.20 \pm 0.42$ & $-1.76 \pm 0.98$ & $<0.001^{*}$ \\
SRS-3C, 1/s & $-1.23 \pm 0.41$ & $-1.78 \pm 0.97$ & $<0.001^{*}$ \\
SRS-2C, 1/s & $-1.21 \pm 0.42$ & $-1.76 \pm 1.00$ & $<0.001^{*}$ \\
GLSRS, 1/s & $-1.21 \pm 0.42$ & $-1.76 \pm 0.96$ & $<0.001$ \\
SRE-4C, 1/s & $1.94 \pm 0.43$ & $2.35 \pm 0.94$ & 0.005 \\
SRE-3C, 1/s & $1.92 \pm 0.43$ & $2.33 \pm 0.95$ & 0.006 \\
SRE-2C, 1/s & $1.91 \pm 0.43$ & $2.32 \pm 0.96$ & 0.005 \\
GLSRE, 1/s & $1.92 \pm 0.43$ & $2.34 \pm 0.95$ & 0.005 \\
SRA-4C, 1/s & $0.78 \pm 0.23$ & $1.04 \pm 0.48$ & 0.001 \\
SRA-3C, 1/s & $0.80 \pm 0.23$ & $1.05 \pm 0.49$ & 0.001 \\
SRA2C, 1/s & $0.81 \pm 0.23$ & $1.06 \pm 0.48$ & 0.001 \\
GLSRA, 1/s & $0.80 \pm 0.23$ & $1.05 \pm 0.49$ & 0.001 \\
\hline
\end{tabular}

Results are shown as mean $\pm \mathrm{SD}$

$4 C-3 C-2 C$ apical four-, three-, and two-chamber views, $G L S$ global longitudinal strain, $G L S R$ global longitudinal strain rate, $L S$ longitudinal strain, $S R A$ late diastolic strain rate, $S R E$ early diastolic strain rate, SRS systolic longitudinal strain rate

*Represents the p value for Mann Whitney U Test

universal acute ischemic injury [32]. They have also suggested that myocardial inflammation could exist without myocyte damage [32]. In a recent MRI-based study, a high rate of subclinical myocardial involvement was reported in patients with COVID-19 infection [11]. The investigators suggested that most patients had myocardial inflammation, regardless of the severity and overall course of the acute disease. Our findings support that patients with mild symptomatic COVID-19 infection may have subclinical myocardial dysfunction. Elevated biomarkers in COVID-19 may only reflect the severity of the disease rather than cardiac involvement. We, therefore, believe that the diagnosis of cardiac involvement can be easily overlooked when the assessment is based on troponin screening alone.

\section{Limitations}

The study should be evaluated in light of some limitations. First, our study population is relatively small, and further studies with greater participation may reveal different results. Second, the study population shows dominance of young age; therefore, the results of this study may not be directly applicable to older populations. Third, recalling bias may exist, we monitored in-home isolation patients mainly via self-reporting. Therefore, some patients may not describe 
their symptoms appropriately. However, the study participants were well educated and cooperative, so this bias may not have substantially affected our main findings. Another limitation is the inability to compare patients with severe infections and those with mild infections.

\section{Conclusions}

COVID-19 is a pandemic, resulting in significant morbidity, mortality, and healthcare costs. Mildly symptomatic COVID-19 patients have subclinical myocardial dysfunction, similar to hospitalized patients according to previous studies (27). LVGLS can be a reliable technique for identifying patients at high risk for long-term cardiovascular complications. Larger studies are necessary to determine the clinical implications of these findings.

\section{Acknowledgements None.}

Author contributions MG: Conceptualization, Data curation, Formal analysis, Project administration, Writing original draft. SI: Methodology, Data curation, Writing original draft, Validation. HA: Methodology, review and editing. OY: Investigation, Validation, Data curation. YA: Investigation, Validation, Writing, Review and Editing.

Funding None.

\section{Declarations}

Conflict of interest All the authors declared that they have no conflict of interest regarding this paper.

Ethical approval Local Ethics Committee Approval Number: 2020 122/2935, Date: 18.12.2020.

Informed consent The informed consent was taken from all patients, and the study was approved by the local ethics committee (No: 2020 122/2935, Date: 18.12.2020). The study was also approved by the Institutional Review Board, and was conducted in accordance with the Helsinki Declaration.

\section{References}

1. Coronavirus disease (COVID-19) pandemic. Numbers at a glance (2021) https://www.who.int/emergencies/diseases/novel-coronavirus-2019. Accessed 5 Jan 2021.

2. Bhatraju PK, Ghassemieh BJ, Nichols M, Kim R, Jerome KR, Nalla AK et al (2020) Covid-19 in critically ill patients in the Seattle region: case series. N Engl J Med 382:2012-2022. https:// doi.org/10.1056/NEJMoa2004500

3. Zhou F, Yu T, Du R, Fan G, Liu Y, Liu Z et al (2020) Clinical course and risk factors for mortality of adult inpatients with COVID-19 in Wuhan, China: a retrospective cohort study. Lancet 395:1054-1062. https://doi.org/10.1016/S0140-6736(20)30566-3

4. Buitrago-Garcia DC, Egli-Gany D, Counotte MJ, Hossmann S, Imeri H, Ipekci AM et al (2020) Occurrence and transmission potential of asymptomatic and presymptomatic SARS-CoV-2 infections: a living systematic review and meta-analysis. PLoS Med 17(9):e1003346. https://doi.org/10.1371/journal.pmed. 1003346

5. Wang Y, Wang Y, Chen Y, Qin Q (2020) Unique epidemiological and clinical features of the emerging 2019 novel coronavirus pneumonia (COVID-19) implicate special control measures. Med Virol 92:568-576. https://doi.org/10.1002/jmv.25748

6. Guan W-J, Ni Z-Y, Hu Y, Liang W-H, Ou C-Q, He J-X et al (2020) Clinical characteristics of coronavirus disease 2019 in China. New Engl J Med 382:1708-1720. https://doi.org/10. 1056/NEJMoa2002032

7. Guo T, Fan Y, Chen M, Wu X, Zhang L, He T et al (2020) Cardiovascular implications of fatal outcomes of patients with coronavirus disease 2019 (COVID-19). JAMA Cardiol 5(7):811-818. https://doi.org/10.1001/jamacardio.2020.1017

8. Long B, Brady WJ, Koyfman A, Gottlieb M (2020) Cardiovascular complications in COVID-19. Am J Emerg Med 38:15041507. https://doi.org/10.1016/j.ajem.2020.04.048

9. Fried JA, Ramasubbu K, Bhatt R, Topkara VK, Clerkin KJ, Horn E et al (2020) The variety of cardiovascular presentations of COVID-19. Circulation 141:1930-1936. https://doi.org/10. 1161/CIRCULATIONAHA.120.047164

10. Shi S, Qin M, Shen B, Cai Y, Liu T, Yang F et al (2020) Association of cardiac injury with mortality in hospitalized patients with COVID-19 in Wuhan, China. JAMA 5(7):802-810. https:// doi.org/10.1001/jamacardio.2020.0950

11. Puntmann VO, Carerj ML, Wieters I, Fahim M, Arendt C, Hoffmann C et al (2020) Outcomes of cardiovascular magnetic resonance imaging in patients recently recovered from COVID19 illness. JAMA Cardiol 5(11):1265-1273. https://doi.org/10. 1001/jamacardio.2020.3557

12. Russo C, Jin Z, Elkind MS, Rundek T, Homma S, Sacco RL et al (2014) Prevalence and prognostic value of subclinical left ventricular systolic dysfunction by global longitudinal strain in a community-based cohort. Eur J Heart Fail 16:1301-1309. https://doi.org/10.1002/ejhf.154

13. Baycan OF, Barman HA, Atici A, Tatlisu A, Bolen F, Ergen $P$ et al (2020) Evaluation of biventricular function in patients with COVID-19 using speckle tracking echocardiography. Int J Cardiovasc Imaging 37:135-144. https://doi.org/10.1007/ s10554-020-01968-5

14. National Health Commission of China. New coronavirus pneumonia prevention and control program [in Chinese]. 6th ed. http://www.nhc.gov.cn/yzygj/s7653p/202002/8334a8326dd94d3 29df351d7da8aefc2/files/b218cfeb1bc54639af227f922bf6b817. pdf. Accessed 2 Feb 2021.

15. Alhazzani W, Møller MH, Arabi YM, Loeb M, Gong MN, Fan E et al (2020) Surviving Sepsis Campaign: guidelines on the management of critically ill adults with coronavirus disease 2019 (COVID-19). Intensive Care Med 46(5):854-887. https:// doi.org/10.1007/s00134-020-06022-5

16. Quinones MA, Otto CM, Stoddard M, Waggoner A, Zoghbi WA (2002) Recommendations for quantification of Doppler echocardiography: a report from the Doppler Quantification Task Force of the Nomenclature and Standards Committee of the American Society of Echocardiography. J Am Soc Echocardiogr 15:167-184. https://doi.org/10.1067/mje.2002.120202

17. Lang RM, Badano LP, Mor-Avi V, Afilalo J, Armstrong A, Ernande L et al (2015) Recommendations for cardiac chamber quantification by echocardiography in adults: an update from the American Society of Echocardiography and the European Association of Cardiovascular Imaging. J Am Soc Echocardiogr 16(3):233-271. https://doi.org/10.1093/ehjci/jev014

18. Nishiga M, Wang DW, Han Y, Lewis DB, Wu JC (2020) COVID-19 and cardiovascular disease: from basic mechanisms 
to clinical perspectives. Nat Rev Cardiol 17(9):543-558. https:// doi.org/10.1038/s41569-020-0413-9

19. Kuck KH (2020) Arrhythmias and sudden cardiac death in the COVID-19 pandemic. Herz 45(4):325-326. https://doi.org/10. 1007/s00059-020-04924-0

20. Kochi AN, Tagliari AP, Forleo GB, Fassini GM, Tondo C (2020) Cardiac and arrhythmic complications in patients with COVID19. J Cardiovasc Electrophysiol 31:1003-1008. https://doi.org/10. $1111 /$ jce. 14479

21. Giustino G, Croft LB, Stefanini GG, Bragato R, Silbiger JJ, Vicenzi M et al (2020) Characterization of myocardial injury in patients with COVID-19. J Am Coll Cardiol 76(18):2043-2055. https://doi.org/10.1016/j.jacc.2020.08.069

22. Ng AC, Delgado V, Bertini M, van der Meer RW, Rijzewijk LJ, Shanks M et al (2009) Findings from left ventricular strain and strain rate imaging in asymptomatic patients with type 2 diabetes mellitus. Am J Cardiol 104:1398-1401. https://doi.org/10.1016/j. amjcard.2009.06.063

23. Mizuguchi Y, Oishi Y, Miyoshi H, Iuchi A, Nagase N, Oki T (2008) The functional role of longitudinal, circumferential, and radial myocardial deformation for regulating the early impairment of left ventricular contraction and relaxation in patients with cardiovascular risk factors: a study with two-dimensional strain imaging. J Am Soc Echocardiogr 21:1138-1144. https://doi.org/ 10.1016/j.echo.2008.07.016

24. Park JH (2019) Two-dimensional echocardiographic assessment of myocardial strain: important echocardiographic parameter readily useful in clinical field. Korean Circ J 49(10):908-931. https://doi. org $/ 10.4070 / \mathrm{kcj} .2019 .0200$

25. Collier P, Phelan D, Klein A (2017) A test in context: myocardial strain measured by speckle-tracking echocardiography. J Am Coll Cardiol 69(8):1043-1056. https://doi.org/10.1016/j.jacc.2016.12. 012

26. Biering-Sorensen T, Biering-Sorensen SR, Olsen FJ, Sengelov M, Jorgensen PG, Mogelvank R et al (2017) Global longitudinal strain by echocardiography predicts long-term risk of cardiovascular morbidity and mortality in a low risk general population: the Copenhagen City heart study. Circ Cardiovasc Imaging 10(3):e005521. https://doi.org/10.1161/CIRCIMAGING. 116.005521

27. Croft LB, Krishnamoorthy P, Ro R, Anastasius M, Zhao W, Buckley S et al (2020) Abnormal left ventricular global longitudinal strain by speckle tracking echocardiography in COVID-19 patients. Future Cardiol. https://doi.org/10.2217/fca-2020-0121 (Ahead of Print)

28. Janus SE, Hajjari J, Karnib M, Tashtish N, Al-Kind SG, Hoit BD (2020) Prognostic value of left ventricular global longitudinal strain in COVID-19. Am J Cardiol 131:134-136. https://doi.org/ 10.1016/j.amjcard.2020.06.053

29. Mitrani RD, Dabas N, Goldberger JJ (2020) COVID-19 cardiac injury: implications for long-term surveillance and outcomes in survivors. Heart Rhythm 17(11):1984-1990. https://doi.org/10. 1016/j.hrthm.2020.06.026

30. Pirzada A, Mokhtar AT, Moeller AD (2020) COVID-19 and myocarditis: what do we know so far? CJC Open 2(4):278-285. https://doi.org/10.1016/j.cjco.2020.05.005

31. Barman H, Atici A, Sahin I, Alici G, Tekin EA, Baycan OF et al (2020) Prognostic significance of cardiac injury in COVID-19 patients with and without coronary artery disease. Coron Artery Dis. https://doi.org/10.1097/MCA.0000000000000914 (Online ahead of print)

32. Bois MC, Boire NA, Layman AJ, Aubry MC, Alexander MP, Roden AC et al (2020) COVID-19-associated non-occlusive fibrin microthrombi in the heart. Circulation 143(3):30-243. https://doi. org/10.1161/CIRCULATIONAHA.120.050754

Publisher's Note Springer Nature remains neutral with regard to jurisdictional claims in published maps and institutional affiliations. 